\title{
Valoración económica de la eficiencia de los sistemas concursales: un análisis empírico internacional
}

\section{Economic valuation of the efficiency of bankruptcy systems: An international empirical analysis}

\section{Carlos López Gutiérrez* . Universidad de Cantabria.}

Myriam García Olalla. Universidad de Cantabria.

Begoña Torre Olmo. Universidad de Cantabria.

\begin{abstract}
RESUMEN La valoración que el mercado realiza de los problemas de insolvencia empresarial se ve condicionada por las características de la legislación concursal, siendo tradicional la distinción entre normativas orientadas a la protección del deudor o de los acreedores. Sin embargo, resulta necesario atender también al propósito perseguido con cada medida concreta establecida en la legislación para tratar de solucionar las dificultades financieras. En el presente trabajo se realiza una aproximación considerando los diferentes tipos de eficiencia de la normativa concursal, el carácter antagónico que, en ocasiones, tienen entre sí, y la valoración que el mercado realiza de cada uno de ellos a través de un análisis internacional en los principales países de la Unión Europea.
\end{abstract}

PALABRAS CLAVE Insolvencia empresarial; Eficiencia legislación concursal; Valoración del mercado.

\begin{abstract}
The market valuation of corporate insolvency problems is conditioned by the attributes of bankruptcy laws, with different codes traditionally being oriented toward protecting the debtor or the creditor. Nevertheless, it is also necessary to address the objective of each concrete measure established in the legislation to try to resolve financial distress. This study carries out an approximation, taking into account the different types of efficiency in bankruptcy regulations, the antagonistic character that, on occasions, exists between them, and the market valuation of each one of these, by way of an international analysis undertaken within the main European Union countries.
\end{abstract}

KEYWORDS Corporate insolvency; Bankruptcy law efficiency; Market Value.

* Autor para correspondencia: Carlos López Gutiérrez. Departamento de Administración de Empresas. Universidad de Cantabria. Avda. de Los Castros s/n, 39005, Santander. Tel. 942 203906, fax 942 201890. Correo-e: carlos.Iopez@unican.es 


\section{INTRODUCCIÓN}

Los problemas de insolvencia empresarial, y los mecanismos de resolución establecidos en la normativa concursal, pueden influir en las decisiones financieras de las empresas y de los inversores, incluso en aquellas situaciones en las que no se prevé la aparición de dificultades financieras. En este sentido, cobra especial relevancia la legislación concursal de cada país, tradicionalmente estudiada en función de que esté orientada hacia la protección del deudor o que proteja los derechos de los acreedores.

En la mayor parte de los casos, los efectos de la ley de insolvencias se han analizado sobre el valor de las acciones de la empresa, a través de estudios de eventos o del comportamiento de los títulos a largo plazo. Además, la mayor parte de estos estudios se han realizado para un único país, lo que no permite contrastar de manera empírica la importancia de las diferencias en la legislación ${ }^{(1)}$. Todos estos trabajos han encontrado una reacción negativa del valor de los títulos en el mercado ante la declaración de insolvencia, como consecuencia de la señal informativa negativa que este hecho supone. No obstante, esa reacción no sólo viene explicada por la manifestación de las dificultades financieras, puesto que los procedimientos legales que van a regular la actividad de la empresa influyen de manera significativa. Así, Gilson et al. (1990), González y González (2000) o López et al. (2005) encuentran que la reacción es diferente en función de la forma en que la empresa va a afrontar sus dificultades, tanto por los propios procedimientos legales como por las transferencias de riqueza que se pueden producir entre los diferentes stakeholders.

En un trabajo reciente, López et al. (2009) realizan una comparación para diferentes países de la UE, en el que comprueban que la orientación de la ley de insolvencias es un factor fundamental en la explicación de los rendimientos a largo plazo de las acciones de las empresas que entran en un procedimiento concursal. En concreto, bajo legislaciones orientadas a la protección de los acreedores encuentran que se produce una mayor pérdida de valor que en los sistemas orientados al deudor, explicada por las características de los propios procedimientos concursales.

No obstante, a pesar de que la comparativa a nivel internacional añade evidencia empírica a la literatura previa, el enfoque sigue centrado en la orientación de la normativa. Sin embargo, podría resultar aún más relevante el propósito del legislador con cada etapa del procedimiento concursal y la aproximación hacia los diferentes tipos de eficiencia aceptados por la literatura, lo que permite analizar en mayor profundidad las razones que explican esa reacción diferencial bajo diferentes sistemas concursales. Desde un punto de vista teórico, se ha planteado el efecto que las características de la ley de insolvencias tienen sobre el comportamiento de todas las empresas de la economía, independientemente de que estén o no atravesando por dificultades financieras. Esta influencia

(1) La mayor parte de los trabajos se centran en el mercado americano, como Aharony et al. (1980), Clark y Weinstein (1983), Gilson et al. (1990), Rimbey et al. (1995), Ferris et al. (1996) o Indro et al. (1999). En otros mercados podemos encontrarnos con Lasfer et al. (1996) para el Reino Unido o González y González (2000) o López et al. (2005) para el mercado español. 
se manifiesta principalmente por los diferentes tipos de eficiencia que puede tener la legislación, y que afectarán de manera diferente a las empresas en función de su situación financiera (Franks et al., 1996; White, 1996; Cornelli y Felli, 1997). En este trabajo se desarrollan dos índices que permiten cuantificar el grado en el que las legislaciones analizadas tratan de lograr determinados tipos de eficiencia, lo que permite realizar una aproximación empírica al problema en un ámbito de estudio en el que estos análisis son inexistentes.

El objetivo principal del trabajo es profundizar en el estudio de las normativas concursales, desde el punto de vista de los efectos que las características de la legislación tienen para las empresas con problemas de insolvencia antes de entrar en un procedimiento concursal. Para ello se analiza una muestra de empresas con problemas de insolvencia en el año inmediatamente anterior a su entrada en un procedimiento concursal.

Las aportaciones del trabajo pueden considerarse desde diferentes puntos de vista. En primer lugar, a la importancia teórica de la orientación de la legislación se le añade la valoración empírica de los distintos tipos de eficiencia de las leyes de insolvencia. Esto permite ampliar la visión del problema, pasando de un enfoque de quién es la parte más beneficiada (orientación al deudor o al acreedor) a otro en el que priman los efectos de las características de la normativa. Además, el trabajo estudia esos efectos para las empresas insolventes antes de su declaración. Esto permite analizar si las repercusiones de determinadas medidas pueden tener un efecto contrario al propósito con el que fueron establecidas cuando la empresa ya está atravesando por dificultades financieras, aspecto propuesto desde el punto de vista teórico pero que no ha sido contrastado de manera empírica hasta ahora.

En segundo lugar, se centra la atención en la valoración realizada por el mercado, lo que aporta un elemento más de discusión a la compleja tarea del diseño de un sistema concursal que resulte eficiente desde diferentes puntos de vista. Así, y siguiendo una vía complementaria de trabajos doctrinales que han abordado esta cuestión, este estudio trata de aportar un elemento objetivo, la valoración realizada por el mercado, que, si bien es cierto no puede tomarse como una medida para determinar qué tipo de normativa es superior, sí que puede arrojar luz sobre ciertos aspectos en los que la literatura no ha conseguido alcanzar conclusiones definitivas.

Por lo que se refiere al ámbito del estudio, el trabajo analiza empresas de Alemania, España, Francia y Reino Unido, países que cubren un amplio espectro de diferentes sistemas concursales. En cada uno de esos países se delimitan las características concretas de sus legislaciones que afectan al valor de la empresa, a través del estudio del grado de eficiencia de la ley, tanto antes como después de la declaración legal de insolvencia. En este sentido, se pone de manifiesto el carácter antagónico que ambos tipos de eficiencia presentan en determinados momentos, con especial atención al efecto ejercido por las medidas establecidas en el procedimiento concursal sobre las empresas con problemas de insolvencia antes de que declaren legalmente su situación, es decir, sobre la eficiencia intermedia, que generalmente ha sido obviada tanto en las investigaciones teóricas como empíricas. 
Las hipótesis que proponemos relacionan la eficiencia de la ley de insolvencia y el valor de las empresas. Nuestra propuesta es que el mercado valorará cada tipo de eficiencia en función de su efecto sobre la situación de las empresas en dificultades financieras. Así, los resultados obtenidos muestran, en primer lugar, que las medidas orientadas hacia la eficiencia ex ante originan una mayor pérdida de valor de mercado para las empresas con problemas de insolvencia antes de su entrada en un procedimiento concursal. Por otro lado, del análisis empírico se desprende que la valoración realizada por el mercado de las legislaciones concursales que traten de maximizar la eficiencia ex post es positiva. En este caso ese efecto dependerá de la situación de la empresa, por lo que la valoración del mercado es positiva siempre que la legislación facilite la continuidad de empresas con mejores perspectivas económicas, y negativa en el caso de que facilite la supervivencia de las empresas que deberían ser liquidadas.

Este estudio en el mercado europeo resulta especialmente relevante en el momento actual puesto que, en la medida en que esta regulación afecte al valor y a las decisiones financieras empresariales, puede actuar como un incentivo al movimiento de capitales dentro de la Unión Europea. Si bien es cierto que las reformas realizadas en los últimos años parecen haber tomado como patrón de referencia los postulados del capítulo 11 norteamericano, no lo es menos que aún existen profundas discrepancias en los procedimientos concursales, lejos todavía de una homogeneización a nivel europeo.

El trabajo se estructura de la manera siguiente: En el epígrafe dos se revisa la literatura y se postulan las hipótesis a contrastar. En el tercer apartado se describen las características de la muestra utilizada para la realización del análisis. Por último, se contrastan los modelos econométricos especificados y se presentan las principales conclusiones de la investigación.

\section{LA EFICIENCIA DE LA LEGISLACIÓN CONCURSAL: IMPLICACIONES SOBRE LA VALORACIÓN DEL MERCADO}

La discusión en torno a la regulación de las situaciones de insolvencia de la empresa se ha planteado tradicionalmente en función de la orientación que debe tener la normativa, es decir, si se debe acentuar la protección en los intereses de los acreedores o en los del deudor.

No obstante, esta perspectiva se ha centrado en el efecto que la legislación concursal tiene sobre las empresas que declaran legalmente su situación de insolvencia, obviando la influencia que esta normativa ejerce sobre las decisiones financieras de todos los partícipes de la empresa, incluso antes de que aparezca ningún síntoma de deterioro de la situación financiera. En este sentido, las investigaciones realizadas en el campo de la insolvencia empresarial ponen de manifiesto la relación existente entre el rendimiento de los títulos de las empresas con problemas de insolvencia y la normativa concursal aplicable. No obstante, resulta necesario profundizar más en el análisis y evaluar las actuaciones concretas previstas en los procedimientos y el propósito de su establecimiento, lo que nos conduce hacia la importancia de los distintos tipos de eficiencia existentes para una determinada normativa concursal, estableciendo la valoración que el mercado realizará de cada uno de ellos. 
La legislación de insolvencia debe tratar de lograr diferentes objetivos que no siempre son compatibles. Por un lado debe decidir qué hacer con la empresa insolvente. Por otro, debe determinar cómo compensar a los acreedores que han visto incumplido su contrato de deuda, sin dejar de maximizar el valor de las empresas que se encuentran en una situación de insolvencia (Hart, 2000). En este sentido, Smith y Strömberg (2005) proponen que la ley de insolvencia debe tratar de maximizar el valor de la empresa como entidad en funcionamiento a lo largo del proceso, pero estableciendo las medidas que faciliten la competencia de los interesados en la reestructuración de la misma. Para conseguirlo, es necesario tener en cuenta tres tipos de eficiencia, que se relacionan con tres periodos que dependen de la información disponible en cada uno de ellos ${ }^{(2)}$ : (Franks et al., 1996; White, 1996): la eficiencia ex ante, que se produce antes de que la empresa tenga dificultades financieras. La eficiencia intermedia, que se produce cuando la empresa tiene dificultades financieras pero aún no ha entrado en el procedimiento legal, situación en la que pueden aparecer problemas en la definición de los derechos de propiedad sobre los activos de la empresa. Por último la eficiencia ex post, que se produce una vez iniciado el proceso, momento en el que hay que decidir qué empresas deben continuar y cuáles deben ser liquidadas.

La eficiencia ex ante trata de evitar que el deudor tome decisiones en contra de los intereses de los acreedores, incentivando el cumplimiento de la devolución de la deuda. Consiste en maximizar el valor obtenido por los acreedores propiciando la aparición de mecanismos para que éstos ejerzan una actividad de control sobre las actuaciones de la empresa (Cornelli y Felli, 1997). De esta forma, la legislación afecta a los incentivos de las distintas partes implicadas al tomar sus decisiones, antes incluso de que exista cualquier indicio de dificultades financieras.

Esto se produce a través de dos efectos. En primer lugar lo que se denomina efecto «castigo» para los directivos, que les estimula a tomar decisiones con el objetivo de evitar tener problemas de insolvencia. El valor futuro de la empresa estará condicionado por el nivel de dedicación o habilidad de la dirección, y el tratamiento aplicado por la ley de insolvencia ejercerá su influencia si incentiva una mejor gestión cuanto más duro sea con la dirección actual. Bajo estas circunstancias, los directivos estarán dispuestos a renunciar a la consecución de sus propios objetivos a cambio de evitar la entrada en el procedimiento de insolvencia (White, 1996). Por lo tanto, los mecanismos que tratan a los directivos de manera más severa proporcionan mayores incentivos a una gestión eficaz y reducen los conflictos de intereses entre el deudor y los acreedores (Aghion et al., 1992).

El segundo efecto se refiere a que la orientación de la ley de insolvencia puede afectar a la financiación de todas las empresas. Davydenko y Franks (2008) y Qian y Strahan (2007) encuentran que el grado de protección a los acreedores es un factor determinante del comportamiento de las entidades financieras a la hora de financiar las empresas de cada país (afecta a las tasas de recuperación, al plazo de las operaciones y a las garantías exigidas). Si la legislación protege especialmente los intereses de los acreedores, puede reducirse el coste de financiación para todas las empresas que operan en el

(2) Según Holmstrom y Myerson (1983), el periodo ex ante comprende el momento antes de que los individuos reciban cualquier tipo de información privada. El intermedio será cuando la información es recibida pero no compartida. Finalmente, el periodo ex post será cuando la información privada es conocida por todos los agentes interesados. 
mercado (Qian y Strahan, 2007; Bae y Goyal, 2004). Las expectativas de recuperación de los acreedores en caso de problemas de insolvencia influyen en sus decisiones y, por lo tanto, contribuyen a determinar la eficiencia ex ante de la normativa. Además, resultan también de gran importancia los incentivos para que el acreedor ejerza una actividad de vigilancia y control sobre la actuación de los directivos de la empresa ${ }^{(3)}$ (Djankov, McLiesh y Shleifer, 2007).

Independientemente del nivel de eficiencia ex ante del procedimiento, resulta inevitable la aparición de problemas de insolvencia en determinadas empresas. En este sentido, la eficiencia intermedia trata de maximizar el valor de las empresas con dificultades financieras antes de que declaren su situación de insolvencia. En esta fase pueden aparecer problemas por decisiones subóptimas de inversión, debido a la existencia de imperfecciones en los mercados de capitales, tales como la asimetría en la información y los costes de agencia. Es decir, puede ocurrir que no todos los proyectos rentables sean llevados a cabo (subinversión) o que se realicen proyectos excesivamente arriesgados, con un valor actual neto negativo (sobreinversión) ${ }^{(4)}$. Estos problemas pueden verse agravados en las empresas con dificultades financieras antes de que se realice la declaración de insolvencia. Los problemas de subinversión se agravan porque los accionistas y directivos no tendrán incentivos para realizar proyectos de inversión rentables, si con ello no reducen la probabilidad de quiebra. Esto ocurre porque este tipo de proyectos reduce la variabilidad de los rendimientos de la empresa, mejorando únicamente la situación de los acreedores (White, 1996). Por otra parte, también los problemas de sobreinversión se pueden ver incrementados en las empresas con dificultades financieras, ya que los directivos tendrán fuertes incentivos para llevar a cabo inversiones excesivamente arriesgadas. Si el proyecto finaliza con éxito se consigue evitar, o al menos retrasar, la entrada en el procedimiento de insolvencia, mientras que si el proyecto fracasa son los acreedores los que soportan el $\operatorname{coste}^{(5)}$.

A estos problemas se une el incentivo a retrasar en lo posible la entrada en el procedimiento. Por un lado, si la potestad de iniciar el procedimiento corresponde al deudor, tratará de retrasarla lo más posible ${ }^{(6)}$. Si, como es más común, son los acreedores los que pueden solicitar el inicio, el deudor tendrá un fuerte incentivo para ocultar su verdadera situación, lo que también llevaría a retrasos y a la aparición de costes adicionales que reducen el valor de la empresa insolvente. Sin embargo, los sistemas que mantienen la empresa bajo el control de la dirección actual reducen este tipo de retrasos ${ }^{(7)}$.

(3) Uno de los principales papeles ejercidos por los acreedores en la economía es el control de las acciones del deudor (Jensen y Meckling, 1976).

(4) Una revisión completa de estos problemas se puede ver en Morgado y Pindado (2003).

(5) La relación entre las características de las legislaciones de insolvencia y los problemas de sobre y subinversión han sido analizados en Pindado et al. (2008).

(6) Los retrasos en el inicio del procedimiento son aún mayores si los directivos tienen una alta participación en la propiedad de la empresa (Bris et al., 2006).

(7) Podemos encontrarnos con empresas que inician el procedimiento con fines estratégicos, por la protección que este tipo de procedimientos les facilitan, y que pueden incluso suponer una ventaja frente a sus competidores (Delaney, 1992; Rose-Green y Dawkins, 2002). 
Por último, una vez que la empresa ha entrado en el procedimiento concursal, nos encontramos con la eficiencia ex post, que se conseguirá cuando como resultado del procedimiento se logre maximizar el valor de la empresa insolvente. Aquellas cuyo valor de liquidación sea mayor que su valor como entidad en funcionamiento deberán ser liquidadas, mientras que el resto maximizarían su valor si se establecen las medidas necesarias para reestructurarlas y se les permite continuar. La existencia de asimetría en la información hace que no se pueda observar de manera inequívoca cuál es la situación real de la empresa, por lo que se pueden cometer dos tipos de errores: por un lado se pueden reestructurar empresas que por su situación deberían haber sido liquidadas (error tipo I); por otro lado, se pueden liquidar empresas cuyo valor como entidad en funcionamiento es mayor que su valor de liquidación (error tipo II). Los inversores conocen los flujos de caja generados en el pasado, pero sólo pueden realizar predicciones acerca de su comportamiento en el futuro. Además, los distintos stakeholders están incentivados a presentar la información más conveniente para sus intereses, por lo que resulta extremadamente difícil determinar cuál es la verdadera situación de la empresa ${ }^{(8)}$.

La existencia de información incompleta, la distinta aversión al riesgo de las partes implicadas y la aparición de costes de transacción, pueden provocar que un sistema que busque maximizar la eficiencia ex ante lleve a la liquidación de empresas cuyo valor en funcionamiento sea mayor que el de liquidación y, por lo tanto, hacer que aparezcan ineficiencias ex post en el proceso. En definitiva, en el desarrollo de una ley concursal se intenta lograr un equilibrio entre ambos tipos, planteándose tal grado de complejidad que provoca la convivencia de legislaciones tan dispares entre los distintos países, siempre teniendo en cuenta la estrecha relación mantenida entre el grado de eficiencia y la orientación de la propia legislación concursal (White, 1996).

Con este planteamiento, el objetivo del trabajo es analizar la valoración que el mercado realiza de las características que presentan las legislaciones concursales desde estos dos puntos de vista. Con el objeto de delimitar claramente la influencia de la legislación concursal, el estudio se centra en las implicaciones observadas para las empresas que han entrado formalmente en un procedimiento de insolvencia, por lo que se verán afectadas por problemas en la eficiencia intermedia y ex post de las legislaciones concursales.

Tradicionalmente, se han planteado los efectos compensatorios de las medidas que tratan de lograr la eficiencia de las legislaciones, relacionado directamente con la orientación que la legislación concursal presenta. Así, se puede plantear que los costes más importantes, y en los que se debe centrar la mayor atención del legislador, son los que se producen por ineficiencias ex ante del proceso, pues afectan a todas las empresas, independientemente de que atraviesen o no por dificultades financieras (White, 1996). No obstante, una legislación centrada básicamente en la reducción de este tipo de costes puede provocar la liquidación de empresas insolventes económica-

(8) Incluso compartiendo las mismas predicciones sobre la generación futura de flujos de caja, pueden existir conflictos sobre la mejor manera de resolver las dificultades financieras. Hay que tener en cuenta que diferentes políticas de reestructuración distribuyen la riqueza entre accionistas, directivos y acreedores de manera distinta (Wruck, 1990). 
mente viables, que incrementarían las ineficiencias ex post del procedimiento (Povel, 1999; Franks et al., 1996).

Sin embargo, el estudio de los efectos negativos que la mejora en la eficiencia ex ante puede tener sobre la ex post, y viceversa, no considera la importancia que ambas actuaciones pueden tener sobre la eficiencia intermedia, y los costes que se pueden originar. Si nos centramos en los efectos de la legislación concursal sobre las empresas con problemas de insolvencia, podemos encontrarnos con que algunos aspectos dirigidos a mejorar el comportamiento de los directivos favorecen la aparición de decisiones oportunistas que reducen el valor de la empresa, cuando ésta ya se encuentra en dificultades financieras.

En particular, las políticas concursales más duras con los directivos, que en principio se postulan como medidas que mejoran la eficiencia ex ante, ejercerán una influencia negativa sobre el valor de las empresas que se encuentran en dificultades financieras. Aunque en estos casos el número de empresas insolventes se reduce, los costes antes de la entrada en el procedimiento se incrementan considerablemente en las empresas que tienen dificultades financieras. A esto hay que añadir los incentivos a retrasar la entrada en el procedimiento, lo que significa un perjuicio tanto para los acreedores, que ven reducida su capacidad de cobro, como para la propia empresa, al disminuir las posibilidades de supervivencia.

La falta de atención hacia las ineficiencias en la fase intermedia podría estar detrás de la poca capacidad de recuperación que han presentado los distintos procedimientos de reestructuración (Couwenberg, 2001) ${ }^{(9)}$. Generalmente, el escaso éxito de estos mecanismos suele explicarse por el alto grado de deterioro con el que las empresas entran en los procedimientos de reestructuración. Esto puede verse agravado por la aparición de decisiones subóptimas de inversión tomadas por los directivos de las empresas con problemas de insolvencia, lo que haría ineficaz el establecimiento de un mecanismo de reestructuración, incluso diseñado de manera eficiente en un sentido ex post. Además, los procedimientos severos con el deudor incentivan la ocultación de información por su parte. Por lo tanto, nos encontramos con un efecto compensación entre la eficiencia ex ante y la intermedia, que dificulta la definición de una normativa concursal eficiente desde un punto de vista global.

De esta forma, las disposiciones incluidas en la legislación con el objetivo de mejorar la eficiencia ex ante, que generalmente tratarán de manera severa al deudor, suponen la aparición de costes en la fase intermedia, por lo que el mercado valorará negativamente este tipo de medidas cuando se trate de empresas que, aunque no hayan realizado una declaración legal, se encuentren en una situación de dificultades financieras. Esto hace que resulte complejo armonizar las actuaciones, no sólo para evitar efectos compensatorios entre la eficiencia ex ante y ex post, que son los más analizados, sino también con los efectos a priori para todas aquellas empresas con problemas de insolvencia antes de tener que realizar la declaración legal de su situación.

(9) Además, el tipo de legislación concursal afecta a las tasas de recuperación dentro del procedimiento, lo que puede ser visto como una medida indirecta de la eficiencia ex post del proceso (Blazy, Petey y Weill, 2009). 
Considerando esta situación, la primera hipótesis que se propone es la siguiente:

Hipótesis 1: «La existencia de medidas orientadas hacia la eficiencia ex ante de la legislación concursal origina una mayor pérdida de valor de mercado para las empresas que ya sufren problemas de insolvencia».

Además, una vez que la empresa entra en el procedimiento, es preciso señalar que la influencia de las medidas orientadas a mejorar la eficiencia ex post no puede ser evaluada de manera global y dependerá del tipo de empresas a las que se apliquen, y de la existencia de filtros efectivos para ello. Así, las actuaciones que conducen a la continuidad de la empresa mejoran la eficiencia ex post siempre que sean económicamente viables, mientras que la empeoran si permiten la supervivencia de empresas que deberían ser liquidadas.

De esta forma, la segunda hipótesis se compone de dos sub-hipótesis estrechamente relacionadas, puesto que la valoración realizada por el mercado de las legislaciones concursales que traten de maximizar la eficiencia ex post será positiva bajo ambas propuestas:

Hipótesis 2.a: «La valoración del mercado será positiva si la legislación facilita la continuidad de empresas que el mercado percibe con mejores perspectivas o mayores probabilidades de éxito».

Hipótesis 2.b: «La valoración del mercado será negativa si la legislación facilita la continuidad de empresas que el mercado percibe con peores perspectivas o pocas probabilidades de éxito».

\section{CARACTERÍSTICAS Y COMPOSICIÓN DE LA MUESTRA}

Para el contraste empírico se ha construido una muestra formada por 199 empresas cotizadas en los mercados de capitales, que han entrado en un procedimiento concursal entre los años 1990 y 2002 en Alemania ${ }^{(10)}$, España, Francia y Reino Unido, países con diferencias significativas en sus sistemas concursales (Franks, Nyborg y Torous, 1996; Kaiser, 1996). La identificación ha sido especialmente difícil y problemática, puesto que no ha sido posible encontrar ningún registro de este tipo de situaciones en algunos de los países que se incluyen en el estudio. De esta forma, se ha procedido a identificar esas empresas por las noticias publicadas en diferentes diarios de cada uno de los países, utilizando la base de datos de noticias proporcionada por Factiva. Se excluyen del estudio las empresas financieras, con regulación propia en caso de producirse un problema de insolvencia.

La composición de la muestra y su distribución por años se reflejan en la Tabla 1:

(10) Hay que tener en cuenta la profunda modificación que la ley concursal alemana ha sufrido en 1999, hecho que se ha considerado a la hora de realizar los análisis pertinentes para evitar distorsiones en los resultados obtenidos. 


\section{TABLA 1}

DisTRIBUCIÓN DE LA MUESTRA POR PAÍSES Y AÑo

\begin{tabular}{|c|c|c|c|c|c|}
\hline Año & Alemania & España & Francia & Reino Unido & TOTAL \\
\hline 1990 & 0 & 0 & 0 & 13 & 13 \\
1991 & 0 & 1 & 0 & 13 & 14 \\
1992 & 1 & 5 & 1 & 9 & 16 \\
1993 & 2 & 4 & 2 & 4 & 12 \\
1994 & 3 & 2 & 0 & 2 & 7 \\
1995 & 0 & 1 & 1 & 3 & 5 \\
1996 & 3 & 2 & 4 & 2 & 11 \\
1997 & 2 & 1 & 3 & 2 & 8 \\
1998 & 2 & 1 & 1 & 4 & 8 \\
1999 & 4 & 0 & 2 & 6 & 12 \\
2000 & 4 & 0 & 4 & 4 & 12 \\
2001 & 21 & 0 & 7 & 6 & 34 \\
2002 & 31 & 1 & 9 & 74 & 47 \\
\hline TOTAL & 73 & 18 & 34 & & 199 \\
\hline
\end{tabular}

La elección del periodo muestral viene condicionada por las características del estudio realizado. Así, resulta necesario realizar el estudio con un periodo de tiempo amplio (1990-2002), de tal forma que la muestra recoja situaciones de insolvencia en distintas fases del ciclo económico en cada uno de los países, para evitar que los resultados se vean afectados por una coyuntura económica determinada. Esto hace que no se incluyan periodos más recientes, debido a los cambios legislativos que se han producido desde el año 2002 (como la entrada en vigor del nuevo procedimiento en el Reino Unido en 2003 y en España en 2004). Este hecho podría distorsionar los resultados, al tener que considerar dos nuevas normativas contando sólo con un número reducido de años para el análisis ${ }^{(11)}$.

Las cotizaciones de los títulos proceden de Datastream, mientras que la información específica de cada empresa se ha obtenido de diferentes fuentes (Datastream, Amadeus y Factiva), puesto que nos encontramos con sociedades que, en muchos casos, han desaparecido o, al menos, han dejado de cotizar en la actualidad lo que dificulta la obtención de información para ellas.

(11) Además, el incremento del número de insolvencias a raíz de la crisis desencadenada en 2008 añadiría un sesgo importante a la muestra, puesto que la mayor parte de las insolvencias bajo estos dos nuevos sistemas se producirían en este periodo. 


\section{4.- ANÁLISIS EMPÍRICO Y RESULTADOS OBTENIDOS}

\section{1.- ÍNDICES DE EFICIENCIA DE LA LEY CONCURSAL}

Para el contraste de las hipótesis propuestas es necesaria una medida cuantitativa del grado en el que cada legislación trata de mejorar los distintos tipos de eficiencia, por lo que se han elaborado índices específicos para las legislaciones concursales de los distintos países. Se ha optado por seguir la metodología aplicada en diversos trabajos que, en algunos casos sin estar centrados en problemas de insolvencia, han tratado de cuantificar el grado de protección de determinadas legislaciones (La Porta et al., 1998; Pistor et al., 2000; La Porta y López-de-Silanes, 2001; Djankov et al., 2008; López et al., 2009). En todos ellos se analizan diferentes variables, dando valores comprendidos entre 1 y 0 en función de la existencia o no de cada medida en la normativa analizada, agregando después todos los valores para obtener un indicador global de cada normativa.

De esta forma, se desarrollan dos índices distintos: el primero que analiza las características de las legislaciones concursales relacionadas con el comportamiento ex ante y el segundo que analiza las disposiciones relacionadas con las posibilidades de continuidad de la empresa. Para ello, se han analizado las legislaciones concursales vigentes en los países objeto de estudio a lo largo de todo el periodo muestral, identificando aquellas características que se consideran más relevantes a la hora de tratar de lograr cada uno de los objetivos propuestos ${ }^{(12)}$. Con el objeto de facilitar su comprensión, en el anexo se presenta una tabla que describe las medidas recogidas en cada legislación en referencia a las variables utilizadas para desarrollar en índice ${ }^{(13)}$.

En el desarrollo del primer índice, Índice de eficiencia ex ante, se incluyen aquellas características que traten de incentivar una mejor gestión por parte de los directivos de todas las empresas mediante mecanismos de castigo o recompensa. En primer lugar se considera quién puede solicitar el inicio del proceso, existiendo tres alternativas: los acreedores, cualquier parte interesada en el proceso (la propia empresa, los acreedores, los trabajadores, el tribunal, etc.) o el deudor. En segundo lugar se incluye quién decide si el procedimiento de reestructuración puede continuar o si la situación de la empresa fuerza su liquidación. Nos encontramos con tres alternativas: que sean los acreedores, directamente o a través de su representante, que sea el tribunal o que sea el propio deudor. La tercera característica es quién gestiona la empresa dentro del procedimiento. Aquí tenemos cinco alternativas: la más favorable al acreedor es que el control de la empresa sea asumido por un administrador externo; después tenemos que en determinados casos se permite la continuidad del deudor (como en la nueva ley alemana en caso

(12) En ambos índices se ha incluido una columna adicional que recoge el valor que tomarían en el caso de la Ley Concursal Española de 2003. A pesar de no estar vigente en el periodo muestral, esto permite observar cuál es la situación actual de nuestra legislación y ver cómo se puede ver afectada por las implicaciones de este trabajo.

(13) En el desarrollo de estos índices se han consultado tanto los textos legislativos de cada país como diversas publicaciones especializadas que analizan cada uno de ellos y sus implicaciones financieras. En concreto, para una revisión de los procedimientos concursales que aquí se presentan se puede consultar Campbell (1992); García Villaverde (1993); Stilpon (1994); Rajak et al. (1995); Kaiser (1996); Franks et al. (1996); Brown (1996); Espina (1999); Mokal (2004). 
de insolvencia inminente); la tercera posibilidad es que lo más común es la continuidad del deudor, aunque en determinadas circunstancias pueda ser sustituido por un administrador (como en el caso francés); la cuarta es que el deudor se mantenga al frente de la empresa, aunque actuando bajo supervisión de una administrador que debe autorizar determinadas operaciones; finalmente tendríamos que el deudor tenga un control completo sobre la empresa. La última característica es la existencia de violaciones de la regla de prioridad absoluta en el pago de los créditos, bien a favor de acreedores privilegiados como el Estado o a favor de los accionistas. Esto perjudica a los acreedores que ven como deudas de inferior prioridad se ven satisfechas mientras que en sus créditos se produce una reducción. Numerosos autores han defendido su utilidad como mejora de las decisiones antes de la entrada en el procedimiento, tanto en lo que se refiere a la declaración en el momento más adecuado (Baird, 1991; Povel, 1999; Berkovitch e Israel, 1999) como a la reducción de problemas de subinversión en la empresa (White, 1989; Gertner y Scharfstein, 1991).

Cada característica tomará un valor máximo de 1 cuando la ley incluya una medida de mejora de la eficiencia ex ante, mientras que en caso contrario tomará el valor mínimo de 0 . No obstante, con el objetivo de que los índices reflejen de manera precisa las diferencias entre cada normativa, en aquellas características que lo requieren se han establecido puntuaciones intermedias (Pistor et al., 2000; Djankov et al., 2008; López et al., 2009). Las puntuaciones en cada una de las características analizadas y la valoración de cada una de las legislaciones se puede observar en la Tabla 2:

TABLA 2

ÍNDICE DE EFICIENCIA EX ANTE

\begin{tabular}{|c|c|c|c|c|c|c|c|}
\hline & Descripción & $\begin{array}{c}\text { Alemania } \\
1\end{array}$ & $\begin{array}{c}\text { Alemania } \\
2\end{array}$ & España & Francia & $\begin{array}{l}\text { Reino } \\
\text { Unido }\end{array}$ & $\begin{array}{l}\text { España } \\
\text { (LC 2003) }\end{array}$ \\
\hline $\begin{array}{l}\text { Potestad de } \\
\text { declaración }\end{array}$ & $\begin{array}{l}\text { Quién tiene la capacidad de iniciar el } \\
\text { procedimiento }\end{array}$ & 0 & 0,5 & 0 & 0,5 & 1 & 0,5 \\
\hline $\begin{array}{l}\text { Decisión } \\
\text { inicial }\end{array}$ & $\begin{array}{l}\text { Quién toma la decisión de iniciar un } \\
\text { proceso de reestructuración o uno de } \\
\text { liquidación }\end{array}$ & 0,5 & 1 & 0.5 & 0.5 & 1 & 0,5 \\
\hline $\begin{array}{l}\text { Gestión } \\
\text { durante } \\
\text { procedimiento }\end{array}$ & $\begin{array}{l}\text { Quién es responsable de la gestión de } \\
\text { la empresa mientras el procedimiento } \\
\text { esté en marcha }\end{array}$ & 0,25 & 0,75 & 0.25 & 0.5 & 1 & 0,25 \\
\hline $\begin{array}{l}\text { Violaciones de } \\
\text { la } A P R\end{array}$ & $\begin{array}{l}\text { Si no se respeta el orden de prioridad } \\
\text { de cobro de cada uno de los créditos }\end{array}$ & 1 & 1 & 0 & 0 & 1 & 0 \\
\hline & Total & 1,75 & 3,25 & 0,75 & 1,5 & 4 & 1,25 \\
\hline \multicolumn{8}{|c|}{$\begin{array}{l}\text { Potestad de declaración: } 1 \text { - Acreedores; 0,5- Deudor } 0 \text { acreedores; } 0 \text { - Deudor. } \\
\text { Decisión Inicial: } 1 \text { - Acreedor o representante; 0,5- Tribunal; 0- Deudor. } \\
\text { Gestión Durante procedimiento: } 1 \text { - Administrador externo; 0,75 - Administrador (preferente) o deudor; 0,5 Deudor (preferente) } \\
0 \text { administrador; 0,25 - Deudor (capacidad de decisión limitada); } 0 \text { - Deudor. } \\
\text { Violaciones APR: } 1 \text { - No; 0- Sí. }\end{array}$} \\
\hline
\end{tabular}

Fuente: Elaboración propia. 
Cuanto mayor sea el índice, mayores incentivos establece la legislación para mejorar las decisiones ex ante de todas las empresas, sean insolventes o no. Sin embargo, como ya hemos analizado, las medidas establecidas para mejorar la eficiencia ex ante pueden ocasionar un agravamiento de los costes para las empresas con dificultades financieras, puesto que un tratamiento más severo de los directivos, tradicionalmente postulado para la mejora de la eficiencia ex ante, provocará mayores problemas de sobre y subinversión, además de incentivar a los directivos para tratar de dilatar lo más posible la entrada en el procedimiento, siempre que la norma les confiera capacidad para hacerlo.

Como se puede observar, el índice toma un mayor valor en el caso del procedimiento concursal del Reino Unido, en el que prácticamente no se deja margen de actuación al deudor, con el objetivo de incentivar mejoras en el comportamiento ex ante. En el extremo opuesto se encuentra la suspensión de pagos española, y en menor medida el procedimiento francés, que conceden mayor capacidad de decisión al deudor, lo que podría ocasionar comportamientos menos eficientes a priori. Por lo que se refiere al caso alemán, se puede observar que la reforma ha incluido medidas tratando de mejorar la eficiencia ex ante del anterior procedimiento.

Una vez que la empresa declara su situación de insolvencia toma importancia el segundo índice, Índice de continuidad, que mide las posibilidades que establece el procedimiento para conseguir la continuidad de la empresa ${ }^{(14)}$. En su construcción se incluyen características relacionadas con el plan de reestructuración, tales como los acreedores a los que afecta (es decir, si hay algún grupo de acreedores que no responden a lo acordado en el plan), o los mecanismos establecidos para su aprobación, que están directamente relacionados con las posibilidades de continuidad de la empresa (en que hay tres posibilidades: que sean los acreedores los que deciden; que la decisión pueda ser compartida con otras partes, principalmente el tribunal; o que el plan necesite la aprobación del deudor). Además, es necesario incluir dos medidas no relacionadas estrictamente con el plan, pero que resultan definitivas a la hora de evaluar las posibilidades de supervivencia. La primera de ellas es la suspensión de las ejecuciones, que supone evitar que los acreedores hagan efectivas sus garantías fuera del procedimiento. Esta medida puede mejorar el comportamiento de las empresas que consigan salir con éxito del procedimiento (Jayaraman et al., 2001). La suspensión automática de las ejecuciones supone de hecho que la entrada en el procedimiento actúa como una protección frente a los acreedores. En este caso tenemos cuatro posibilidades: que no exista esta suspensión; que la suspensión sólo afecte a los acreedores junior; que afecte a todos los acreedores, pero limitada en el tiempo por ley; y que afecte a todos los acreedores de la empresa. La segunda característica es la posibilidad de emitir deuda de mayor prioridad. Con ello, se pretende facilitar a la empresa la obtención de los fondos necesarios para mantener en funcionamiento la actividad el tiempo necesario para llegar a un acuerdo con sus acreedores y evitar su liquidación. Esta medida aumenta las posibilidades de supervivencia, contribuye a la reducción de la asimetría de la información entre los acreedores y el

(14) Este tipo de mecanismos deberían orientarse a encontrar una solución más eficiente tratando de no incluir distorsiones en los incentivos establecidos desde el punto de vista ex ante (Webb, 1991), aunque en la práctica éste se ha mostrado como un equilibrio difícil de conseguir. 
deudor y puede reducir la duración y los costes del procedimiento (Elayan y Meyer, 2001; Dahiya et al., 2003; Chatterjee et al., 2004). Hay que tener en cuenta que la posibilidad de emisión de deuda con carácter prioritario puede suponer la reducción de problemas de racionamiento de crédito en las empresas insolventes, pero podría incrementar el coste de capital para todas las empresas a priori. Estas dos últimas son medidas que no presentan tanta relación con las actuaciones futuras contenidas en el plan, pero que tienen un valor fundamental para conseguir mantener la viabilidad del negocio a lo largo de la duración del proceso, hecho fundamental para que la empresa pueda salir del procedimiento con un acuerdo de continuidad.

$\mathrm{Al}$ igual que el caso anterior, en el desarrollo de este segundo índice cada una de las variables toma un valor máximo de 1 si la legislación facilita la continuidad de la empresa o 0 en el caso opuesto, permitiendo también puntuaciones intermedias en aquellas características que lo requieren. De esta forma, un mayor valor del índice implica mayores facilidades establecidas por la normativa para conseguir la supervivencia de la empresa. El índice para cada uno de los países lo podemos observar en la Tabla 3:

TABLA 3

ÍNDICE DE CONTINUIDAD

\begin{tabular}{|c|c|c|c|c|c|c|c|}
\hline & Descripción & $\begin{array}{c}\text { Alemania } \\
1\end{array}$ & $\begin{array}{c}\text { Alemania } \\
2\end{array}$ & España & Francia & $\begin{array}{l}\text { Reino } \\
\text { Unido }\end{array}$ & $\begin{array}{l}\text { España } \\
\text { (LC 2003) }\end{array}$ \\
\hline $\begin{array}{l}\text { Afecta } \\
\text { Acreedores } \\
\text { Garantizados }\end{array}$ & $\begin{array}{l}\text { Si hay acreedores que no se ven } \\
\text { sujetos a lo acordado en el plan de } \\
\text { insolvencia }\end{array}$ & 0 & 1 & 0 & 1 & 0 & 1 \\
\hline $\begin{array}{l}\text { Aprobación del } \\
\text { plan }\end{array}$ & $\begin{array}{l}\text { Mecanismos y agentes implicados en } \\
\text { la aprobación del plan }\end{array}$ & 0 & 0,5 & 1 & 0,5 & 0 & 0 \\
\hline $\begin{array}{l}\text { Suspensión de } \\
\text { Ejecuciones }\end{array}$ & $\begin{array}{l}\text { Si existe una suspensión de } \\
\text { ejecución de los créditos durante el } \\
\text { procedimiento }\end{array}$ & 0,5 & 0,75 & 0,5 & 1 & 0 & 0,75 \\
\hline Deuda posterior & $\begin{array}{l}\text { Si se permite la emisión de deuda } \\
\text { durante el procedimiento que tenga } \\
\text { carácter prioritario sobre la existente }\end{array}$ & 0 & 1 & 1 & 1 & 0 & 1 \\
\hline & Total & 0,5 & 3,25 & 2,5 & 3,5 & 0 & 2,75 \\
\hline \multicolumn{8}{|c|}{$\begin{array}{l}\text { Afecta acreedores garantizados: } 0 \text { - No; } 1 \text { - Sí. } \\
\text { Aprobación del plan: } 0 \text {-Acreedores; } 0,5 \text { - Con intervención del tribunal; } 1 \text { - Aprobación del deudor requerida. } \\
\text { Suspensión de Ejecuciones: } 0 \text { - No: } 0,5 \text { No para acreedores garantizados; } 0,75 \text { - Con límites temporales para los acreedores } \\
\text { garantizados; } 1 \text { - Todos los acreedores. } \\
\text { Deuda posterior: } 0 \text { - No; } 1 \text { - Sí. }\end{array}$} \\
\hline
\end{tabular}

Fuente: Elaboración propia.

En este caso, la legislación que pone menos facilidades para la continuidad de la empresa insolvente es la del Reino Unido, tanto por la protección de la empresa frente a los acreedores como por las medidas que pudieran facilitar su continuidad una vez iniciado el procedimiento. Algo similar ocurre que el antiguo procedimiento de Alemania, en el que el grado de protección a los acreedores garantizados suponía, de hecho, un serio obstáculo a la continuidad de las operaciones de la empresa con dificultades (Kaiser, 
1996). Por otro lado nos encontramos con el procedimiento de Francia y el nuevo de Alemania, que incluyen una serie de medidas destinadas a facilitar la reorganización de la empresa. En el caso de Francia hay que tener en cuenta el gran protagonismo que toma el tribunal en todo el proceso, por lo que en muchas ocasiones este tiene que tomar una decisión entre lograr una eficiencia social, el manteamiento del empleo, y una eficiencia financiera, logrando una salida que maximice el valor de la empresa tras el procedimiento (Chopard, Guigou, Fimayer y Blazy, 2007) ${ }^{(15)}$. En el caso de la suspensión de pagos española nos encontramos con una situación intermedia, puesto que por un lado incluye medidas orientadas a la continuidad de la empresa, pero por otro deja fuera de ellas a los acreedores garantizados. Hay que señalar que la eficiencia ex post es prácticamente imposible de conseguir en un entorno de información incompleta, puesto que los incentivos al mantenimiento de la empresa pueden hacer que permanezcan empresas que deberían haber sido liquidadas y viceversa.

Analizando la situación desde el punto de vista de la reforma aprobada en España en 2003, se observa que la nueva Ley Concursal ha modificado la situación desde el punto de vista de los dos índices, mejorando los valores en cada uno de ellos. Por lo que se refiere a las medidas relativas al comportamiento ex ante, se elimina el derecho exclusivo que tenía el deudor para iniciar un procedimiento de reestructuración. En cuanto a las posibilidades de continuidad, se reducen los privilegios de los acreedores garantizados dentro del procedimiento, así como la necesidad de que el plan de reorganización cuente con el apoyo del deudor.

\subsection{MEDIDA DE RENDIMIENTO Y METODOLOGÍA}

La literatura financiera propone como medida más adecuada para el cálculo de los rendimientos a largo plazo componer los rendimientos a corto plazo, generalmente mensuales, para obtener el rendimiento correspondiente al horizonte temporal que se desea analizar (Barber y Lyon, 1997; Kothari y Warner, 1997; Fama, 1998; Lyon et al., 1999; Cowan y Sergeant, 2001). De esta forma, para cuantificar la pérdida de valor experimentada por las empresas con dificultades financieras el año inmediatamente anterior a la declaración legal de insolvencia, aplicamos una estrategia de «comprar y mantener» ${ }^{(16)}$, es decir, para cada empresa de la muestra se calcula el rendimiento obtenido si se hubiera comprado un año antes y vendido el mes de la declaración de insolvencia. El rendimiento se calcula de la siguiente manera:

$$
\mathrm{BHR}_{\mathrm{it}}=\left[\prod_{\mathrm{t}=0}^{\mathrm{T}_{\mathrm{j}}}\left(1+\mathrm{R}_{\mathrm{it}}\right)\right]-1
$$

(15) En este sentido, Luttikhuis (2008) propone que la eficiencia social de la ley de insolvencias no sólo se logra con el mantenimiento del empleo, sino que en este concepto habría que incluir la continuidad de la empresa.

(16) La utilización de este tipo de medidas en el estudio de rentabilidades de empresas con problemas de insolvencia, frente a los estudios clásicos de eventos, no ha sido muy abundante, si bien podemos encontrarlo en trabajos como Clark y Weinstein (1983), Rimbey et al. (1995), Indro et al. (1999) o López et al. (2009). 
donde:

$\mathrm{BHR}_{\mathrm{it}}$ : Rentabilidad proporcionada por una estrategia de «comprar y mantener» durante los $t$ meses antes de la declaración para el título $i$.

$\mathrm{R}_{\mathrm{it}}$ : Rentabilidad del título $i, t$ meses antes de la fecha de la declaración.

$\mathrm{T}_{\mathrm{j}}$ : Mes antes de la declaración en la que se considera la compra de los títulos.

El modelo se estima aplicando la metodología de regresión lineal múltiple con datos de sección cruzada. La estimación se ha realizado aplicando mínimos cuadrados ordinarios (MCO) mediante el estimador robusto que utiliza la corrección de White para controlar los problemas de heterocedasticidad (White, 1980).

La especificación del modelo que proponemos toma la forma siguiente:

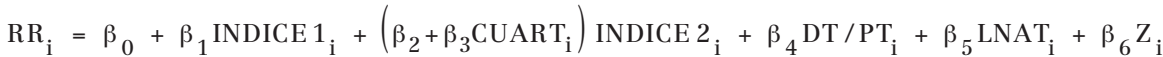

$$
\begin{aligned}
& +\beta_{7} \mathrm{SF}_{\mathrm{i}}+\sum_{\mathrm{j}=1}^{3} \gamma_{\mathrm{j}} \text { SECTOR }+\sum_{\mathrm{k}=1}^{13} \lambda_{\mathrm{k}} \mathrm{AÑO}_{\mathrm{ki}}+\varepsilon_{\mathrm{i}}
\end{aligned}
$$

En la Tabla 4 presentamos la definición de las variables utilizadas:

TABLA 4

\section{VARIABLES INDEPENDIENTES DEL MODELO DE REGRESIÓN}

\begin{tabular}{|l|l|}
\hline \multicolumn{1}{|c|}{ VARIABLE } & \multicolumn{1}{c|}{ DESCRIPCIÓN } \\
\hline$R R$ & $\begin{array}{l}\text { Estimador de riqueza relativa. Cociente del rendimiento a largo plazo de la empresa entre el rendimiento } \\
\text { del mercado en el mismo periodo. }\end{array}$ \\
\hline INDICE1 & Índice que mide el grado de eficiencia ex ante de la legislación concursal \\
\hline INDICE2 & $\begin{array}{l}\text { Índice que mide el grado en el que la legislación concursal facilita la continuidad de las empresas con } \\
\text { problemas de insolvencia }\end{array}$ \\
\hline AdBHR & $\begin{array}{l}\text { Rendimiento a largo plazo ajustado. Rendimiento a largo plazo de la empresa menos el rendimiento del } \\
\text { mercado. }\end{array}$ \\
\hline CUART & $\begin{array}{l}\text { Empresas con mayor rendimiento ajustado. Variable dummy que toma el valor 1 si la empresa pertenece } \\
\text { al primer cuartil de AdBHR y cero en caso contrario. }\end{array}$ \\
\hline LNAT & Tamaño de la empresa. Logaritmo neperiano del activo total. \\
\hline DT/PT & Nivel de endeudamiento. Ratio de la deuda total sobre el pasivo total de la empresa. \\
\hline SF & $\begin{array}{l}\text { Tipo de sistema financiero. Logaritmo neperiano de la importancia de la capitalización bursátil sobre el } \\
\text { GDP entre la importancia de los créditos domésticos al sector privado sobre el GDP. }\end{array}$ \\
\hline Z & $\begin{array}{l}\text { Probabilidad de quiebra. Variable dummy que toma el valor 1 cuando el Z score de Altman clasifica a la } \\
\text { empresa como con alta probabilidad de quiebra (Z < 1.81) y cero en caso contrario. }\end{array}$ \\
\hline SECTOR & $\begin{array}{l}\text { Variables dummy que identifican el sector de actividad de la empresa (Industrial, tecnológico 0 } \\
\text { servicios) }\end{array}$ \\
\hline VNO & Variables dummy que identifican el año (1990-2002) \\
\hline
\end{tabular}


La variable dependiente del modelo refleja el comportamiento de los títulos de la empresa en el mercado en el año anterior a la declaración de insolvencia con respecto al comportamiento del mercado en el mismo periodo. Su cálculo se realiza a través de un estimador de riqueza relativa (RR), utilizado ampliamente en la literatura relativa al análisis de los rendimientos a largo plazo (Ritter, 1991; Loughram y Ritter, 1995; Brav et al., 2000; Jakobsen y Voetmann, 2005) . Su cálculo se realiza de acuerdo con la siguiente expresión:

$$
\mathrm{RR}_{\mathrm{i}}=\frac{1+\mathrm{BHR}_{\mathrm{i}}}{1+\mathrm{BHR}_{\mathrm{M}}}
$$

Donde $\mathrm{BHR}_{\mathrm{i}}$ representa el rendimiento con una estrategia de comprar y mantener de la empresa en el año inmediatamente anterior a la declaración de insolvencia y $\mathrm{BHR}_{\mathrm{M}} \mathrm{el}$ rendimiento del mercado en el mismo periodo. Si este índice toma un valor menor que uno se interpreta como un peor comportamiento de la empresa con respecto al índice de referencia.

Como variables independientes se incluyen los índices relativos a las características de las legislaciones concursales desarrollados en este trabajo. El primero de ellos (INDICE1) centrado en el grado en que cada normativa incentiva mejoras en el comportamiento ex ante del deudor y el segundo (INDICE2) que mide las facilidades que la ley establece para la continuidad de las empresas insolventes. No obstante, para el contraste de la segunda hipótesis resulta necesario incluir una variable interacción del INDICE2, que permitirá analizar el efecto que estas medidas tendrán en función de la situación de la empresa.

De esta forma, resulta necesario dividir la muestra de las empresas insolventes, distinguiendo aquellas que el mercado percibía como con mejores posibilidades de supervivencia en el año antes de la declaración. Esta clasificación no es posible realizarla de manera exacta, por lo que debemos aplicar una variable Proxy que nos permita analizar el efecto diferencial en función de la situación de la empresa y su percepción por el mercado. Una forma de aproximarse a esta cuestión es a través de la reacción previa del mercado antes de la declaración, que puede suponer, en cierta forma, una medida que permita anticipar las probabilidades de quiebra de la empresa (Beaver, 1968; Aharony et al., 1980) ${ }^{(17)}$. Además, Clark y Weinstein (1983) encuentran que los títulos que experimentan una mayor pérdida de valor antes de la declaración corresponden a las empresas con un peor comportamiento una vez iniciado el procedimiento concursal. De esta forma, la magnitud de la pérdida refleja la valoración que realiza el mercado de la situación por la que atraviesa la empresa, y las perspectivas que se tienen sobre su comportamiento en el futuro.

(17) Si los precios reflejan la información que existe en los estados financieros, y a su vez parte de dicha información tiene capacidad para predecir la quiebra de las empresas, es de suponer que dicha información se verá reflejada también en los precios de las acciones (Mora, 1995) 
En su estudio de las fuentes de ganancias para los accionistas en los procedimientos de insolvencia en el mercado norteamericano, Indro et al. (1999) utilizan una medida de rendimiento ajustado a largo plazo para clasificar a las empresas con mejor o peor comportamiento. Así, calculamos el rendimiento a largo plazo ajustado (AdBHR) como la diferencia entre la rentabilidad a largo plazo de los títulos de la empresa menos el rendimiento a largo plazo del mercado, siguiendo la expresión:

$$
\operatorname{AdBHR}_{i t}=\left[\prod_{t=0}^{T_{j}}\left(1+R_{i t}\right)\right]-\left[\prod_{t=0}^{T_{j}}\left(1+R_{M t}\right)\right]
$$

Esta variable así construida nos a va permitir realizar la división de la muestra. De esta forma, aquellas empresas cuyo rendimiento a largo plazo ajustado sea mayor serán las que el mercado anticipa que tienen una mayor probabilidad de afrontar con éxito sus dificultades financieras. Para introducir esta circunstancia en el análisis resulta necesario categorizar esta variable, para evaluar el efecto que el índice que facilita la continuidad de la empresa tiene en función de su situación. Así, dividimos las empresas de la muestra por cuartiles de la variable AdBHR, identificando como las empresas con mayor probabilidad de éxito a las que pertenecen al primer cuartil, e incluyendo una variable dummy (CUART) que toma valor 1 para ellas y cero en caso contrario. Esto nos permite contrastar la reacción del mercado ante el nivel de eficiencia ex post del proceso de manera indirecta, comprobando si los incentivos a la continuidad afectan positivamente a los rendimientos de las empresas con una mejor situación. Así, se incluye un término interacción que implica el producto de dos variables explicativas, el índice que mide las facilidades para la continuidad de la empresa establecidas en cada legislación (INDICE2) y la variable dummy que diferencia a las empresas con mayores probabilidades de éxito (CUART).

En el modelo se incluyen una serie de variables de control, que han sido encontradas en la literatura previa como factores que afectan al rendimiento de los títulos. En primer lugar se introduce el tamaño (LNAT), que se ha mostrado como un factor fundamental en la explicación de la rentabilidad de las acciones de la empresa (Fama y French, 1992). En este caso el tamaño se introduce en el modelo medido por el logaritmo neperiano del activo total, utilizado en diversos trabajos que analizan el rendimiento de los títulos de las empresas con dificultades financieras (Rimbey et al., 1995; Alderson y Betker, 1999; Lopez et al., 2009). En segundo lugar, se incluye el nivel de endeudamiento (DT/PT), porque cuanto mayor es este mayor será el riesgo financiero percibido por los inversores y mayores dificultades tendrá la empresa con problemas para sobrevivir (Gilson et al., 1990; Asquith et al., 1994) ${ }^{(18)}$. Esto hace que el grado de endeudamiento pueda tener una relación negativa con los rendimientos anormales de las empresas con problemas de insolvencia, tal y como encuentran Dawkins y Rose-Green (1998). Para controlar el efecto que puede tener la probabilidad de que la empresa acabe entrando en un procedimiento concursal, se incluye una variable

(18) Además hay que tener en cuenta la relación existente entre la estructura de capital de la empresa y los problemas de insolvencia, no sólo desde el punto de vista de la teoría del trade off sino por el efecto que los problemas de liquidez pueden ejercer sobre el nivel de endeudamiento (Uhrig-Homburg, 2005). 
dummy utilizando el modelo Z score de Altman (1968). Los modelos de predicción realizan una evaluación a priori de las posibilidades de fracaso, siendo su utilidad práctica esa capacidad de anticipación de las dificultades financieras de la empresa (Jiménez, 1996) ${ }^{(19)}$. Así, para cada una de las empresas de la muestra se ha calculado el valor de la $\mathrm{Z}$ en cada uno de los años (Rose-Green y Dawkins, 2002). Las empresas con mayor probabilidad de quiebra, en las que la variable dummy toma el valor de 1 , son aquellas que tienen un valor de la $\mathrm{Z}$ inferior a 1,81. También se incluye una variable que controla por el tipo de sistema financiero (SF), debido a su relación con un diseño apropiado de la ley de insolvencia (Berkovitch e Israel, 1999). Esta variable es medida a través de la importancia de los mercados de capitales con respecto al sector bancario (Levine, 2002). Por último, se incluyen distintas variables dummy para controlar el sector de actividad de la empresa (SECTOR) y variables dummy temporales para controlar el año (AÑO).

En la Tabla 5 se pueden observar los estadísticos descriptivos de todas estas variables utilizadas, así como la matriz de correlaciones entre las variables independientes del modelo:

\section{TABLA 5}

ESTADÍSTICOS DESCRIPTIVOS Y CORRELACIONES

\begin{tabular}{|c|c|c|c|c|c|c|c|}
\hline \multicolumn{6}{|c|}{ Panel 1: Estadísticos descriptivos } & & \\
\hline Variable & $\begin{array}{c}\text { Observa- } \\
\text { ciones }\end{array}$ & Media & $\begin{array}{l}\text { Desviación } \\
\text { Típica }\end{array}$ & Mínimo & Máximo & & \\
\hline $\mathrm{RR}$ & 199 & 0,2453 & 0,2290 & 0,0063 & 1,3338 & & \\
\hline INDICE1 & 199 & 2,9058 & 1,1545 & 0,75 & 4 & & \\
\hline INDICE2 & 199 & 1,8367 & 1,5806 & 0 & 3,5 & & \\
\hline DT/PT & 199 & 0,7279 & 0,3718 & 0,0539 & 4,1222 & & \\
\hline LNAT & 199 & 11,2507 & 1,5265 & 7,6217 & 16,1579 & & \\
\hline Z & 199 & 0,3920 & 0,4894 & 0 & 1 & & \\
\hline SF & 199 & $-0,5302$ & 0,5685 & $-1,6879$ & 0,4997 & & \\
\hline \multicolumn{8}{|c|}{ Panel 2: Matriz de correlaciones } \\
\hline & INDICE1 & INDICE2 & $\begin{array}{c}\text { INDICE2* }^{*} \\
\text { CUART }\end{array}$ & $\mathrm{DT} / \mathrm{PT}$ & LNAT & Z & SF \\
\hline INDICE1 & 1 & & & & & & \\
\hline INDICE2 & $-0,5665$ & 1 & & & & & \\
\hline INDICE2*CUART & $-0,2527$ & 0,3955 & 1 & & & & \\
\hline DT/PT & $-0,0979$ & $-0,2129$ & $-0,0271$ & 1 & & & \\
\hline LNAT & 0,0192 & $-0,0875$ & 0,0235 & 0,0469 & 1 & & \\
\hline Z & $-0,2896$ & 0,0832 & 0,0612 & 0,3092 & 0,1252 & 1 & \\
\hline SF & 0.3999 & -0.4775 & -0.2019 & 0.0682 & -0.1995 & & 1 \\
\hline
\end{tabular}

(19) Los modelos de predicción basados en ratios han sido ampliamente utilizados en la literatura frente a los basados en datos de mercado, no existiendo una gran diferencia en cuanto a la precisión de sus predicciones (Agarwal y Taffler, 2008). 


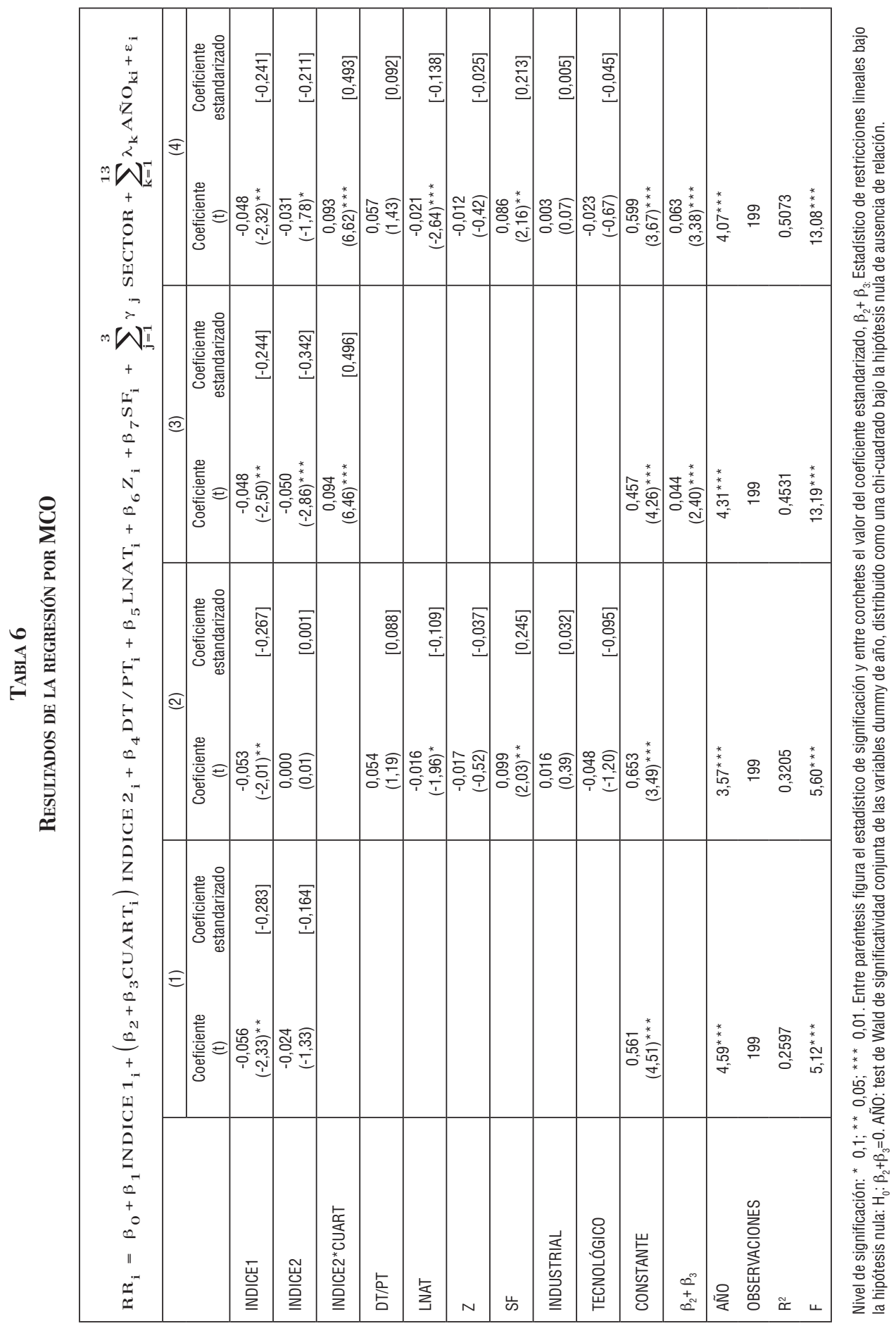




\subsection{Resultados OBTEnidos}

En la Tabla 6 se presentan los resultados obtenidos de la estimación de cuatro especificaciones distintas del modelo propuesto. Los modelos (1) y (2) no incluyen la variable interacción, y evalúan el efecto del INDICE2 de manera global, sin distinguir la situación de las empresas, mientras que este efecto se incluye en los modelos (3) y (4) a través de la variable interacción INDICE2*CUART. En ambos casos se presenta el modelo sólo con los índices y añadiendo el resto de variables de control definidas en el epígrafe anterior.

Para evaluar la significación económica de la orientación de la legislación concursal, se han calculado los coeficientes estandarizados para cada una de las variables del modelo. Esto permite eliminar el efecto escala de los coeficientes estimados, y determinar cuáles son los factores que tienen una mayor influencia sobre la variable dependiente.

Para contrastar las dos hipótesis propuestas debemos interpretar los coeficientes de las variables que incluyen los índices de eficiencia de la legislación de insolvencia. Para evaluar la influencia del primer índice necesitamos contrastar la hipótesis nula $\mathrm{H}_{0}$ : $\beta_{1}=0$. Si rechazamos esta hipótesis nula el coeficiente $\beta_{1}$ es estadísticamente diferente de cero y nos mide la sensibilidad de comportamiento a largo plazo del índice de riqueza relativa de la empresa al grado en que la ley de insolvencias incentiva mejoras en el comportamiento ex ante.

Para el caso del segundo índice debemos analizar dos relaciones. En primer lugar, analizamos el efecto para las empresas que el mercado considera con mejor situación (en las que la variable CUART toma valor 1). Para una correcta interpretación de la variable interacción resulta necesario realizar test de restricciones lineales para contrastar la significatividad de las sumas de coeficientes. En este caso la hipótesis nula será $\mathrm{H}_{0}$ : $\beta_{2}+\beta_{3}=0$. Si esta hipótesis nula es rechazada la suma de los coeficientes refleja la influencia de la orientación de la ley de insolvencias para este grupo de empresas. En segundo lugar, analizamos las empresas consideradas con menor probabilidad de éxito en el procedimiento (en las que la variable CUART toma valor 0). Para evaluar la influencia del segundo índice para este grupo de empresas necesitamos contrastar la hipótesis nula $\mathrm{H}_{0}$ : $\beta_{2}=0$. Si rechazamos esta hipótesis nula el coeficiente $\beta_{2}$ es estadísticamente diferente de cero y nos mide la sensibilidad de comportamiento a largo plazo del índice de riqueza relativa de la empresa al grado en que la ley de insolvencias incentiva la continuidad de las empresas en aquellos casos en que el mercado considera que tienen una peor situación o menor probabilidad de éxito.

De acuerdo con las hipótesis propuestas los signos esperados son los siguientes. La primera hipótesis propone que las medidas orientadas hacia la eficiencia ex ante de la legislación concursal originan un peor comportamiento del valor de mercado para las empresas con problemas de insolvencia, por lo que el signo esperado del coeficiente $\beta_{1}$ será negativo. La hipótesis 2.a defiende que la valoración del mercado será positiva siempre que la legislación facilite la continuidad de empresas con mejores posibilidades de éxito, por lo que el signo esperado de $\left(\beta_{2}+\beta_{3}\right)$ será positivo. Por último, la hipótesis $2 . b$ propone que la valoración del mercado será negativa si la legislación facilita la continuidad de empresas con pocas probabilidades de éxito, por lo que el coeficiente $\beta_{2}$ será negativo. 
Los resultados obtenidos muestran que la variable INDICE1 presenta un coeficiente negativo y estadísticamente significativo a un nivel superior al 95\% en todos los modelos estimados. Esto indica que las legislaciones que establecen fuertes incentivos para un comportamiento eficiente ex ante provocarán una mayor pérdida de valor en las empresas que se encuentran en dificultades financieras, al producirse mayores costes que empeoran la eficiencia intermedia, tal y como propone la hipótesis 1. Los inversores parecen percibir que cuanto mayor sea la exigencia para los directivos y accionistas en el procedimiento concursal ${ }^{(20)}$, mayores incentivos aparecen para la toma de decisiones subóptimas de inversión en empresas con problemas de insolvencia, y mayor probabilidad existe de que el deudor trate de retrasar la entrada en el procedimiento. Por lo tanto, las medidas orientadas a mejorar la eficiencia ex ante tienen una repercusión negativa si consideramos su influencia únicamente sobre las empresas que atraviesan problemas de insolvencia financiera, desde el momento en que se empiezan a manifestar sus dificultades financieras hasta que se realiza la declaración legal de insolvencia.

Este resultado concuerda con la evidencia previa existente hasta el momento. Por un lado, desde el punto de vista de la influencia que el mecanismo de solución de los problemas de insolvencia tiene sobre el rendimiento de los títulos de la empresa. Así, números trabajos han estudiado este problema (Chatterjee et al., 1996; Gilson et al., 1990; González y González, 2000; López et al., 2005), encontrando que afrontar las dificultades financieras dentro de un procedimiento legal supone una reacción del mercado diferenciada con respecto a las soluciones privadas (workouts), lo que se explica por las medidas contenidas en los propios procedimientos concursales. Por otro lado, Lopez et al. (2009) encuentran que las características de la ley de insolvencias ejercen una influencia significativa sobre la reacción del mercado ante las dificultades financieras. Nuestra primera hipótesis aporta evidencia adicional, mostrando la influencia que en concreto tienen las medidas orientadas a mejorar la eficiencia ex ante, a través del deterioro que se produce en la eficiencia intermedia.

Por otro lado, la variable INDICE2 no muestra ninguna influencia sobre la valoración que el mercado realiza de los títulos de estas empresas, tal y como se puede observar en los modelos (1) y (2). No obstante, tal y como hemos comentado, esta ausencia de efecto significativo puede estar enmascarando la repercusión asimétrica que este tipo de medidas puede ejercer en función de las circunstancias de cada una de las empresas, por lo que resulta necesario considerar esto en el análisis empírico a través de una variable interacción.

Los resultados reflejan que las posibilidades de continuidad que establece la legislación ejercen una influencia positiva y significativa a un nivel superior al 1\% en las empresas que pertenecen al grupo de mejor comportamiento en el mercado en el año antes de la declaración, tal y como se observa en el test de restricciones lineales de la suma de coeficientes $\beta_{2}+\beta_{3}$. Por lo tanto, los inversores valoran de manera positiva este tipo de mecanismos incluidos en el procedimiento si perciben que la empresa es económicamente

(20) Lo que incrementará la eficiencia ex ante, es decir, contribuye a la mejora en la gestión de las empresas antes de la existencia de dificultades financieras. 
rentable, tal y como hemos propuesto en la hipótesis 2.a. No obstante, la eficiencia ex post de la legislación concursal se consigue evitando dos tipos de errores, tipo I y tipo II. En este sentido, los resultados también muestran claramente cómo el mercado valora negativamente que las legislaciones permitan que continúen empresas que deberían ser liquidadas, error tipo I, tal y como hemos propuesto en la hipótesis 2.b, al ser el coeficiente $\beta_{2}$ negativo y estadísticamente significativo.

Estos resultados aportan evidencia adicional a lo obtenido por Lopez et al. (2009), que encuentran que bajo legislaciones de insolvencia orientadas al deudor se produce una menor pérdida de valor en el mercado que bajo aquellas más centradas en la protección de los acreedores. No obstante, si consideramos el problema desde el punto de vista de la eficiencia de la normativa esta visión puede resultar limitada, al no diferenciar el efecto en función de la situación de la empresa. De esta forma, de acuerdo con nuestros resultados, la valoración positiva del mercado no se produce en el caso de legislaciones que favorecen la continuidad de todas las empresas, sino que ésta aparece para aquellos mecanismos que persiguen lograr la eficiencia ex post del proceso. Esto se consigue permitiendo el mantenimiento de las empresas cuyo valor percibido por el mercado es superior a su valor de liquidación, por lo que además se produce una valoración negativa de este tipo de medidas aplicadas a empresas con un peor comportamiento, cuya continuidad supondría la aparición de ineficiencias a posteriori.

Por lo que se refiere a las variables específicas de cada empresa, el tamaño (LNAT) ejerce una influencia negativa y estadísticamente significativa, lo que puede explicarse porque las empresas de mayor tamaño presentan una mayor complejidad en su estructura de capital (Betker, 1995), además de existir un mayor número de partes implicadas en las negociaciones en caso de insolvencia (Eberhart et al., 1990; Franks y Torous, 1994), lo que puede ocasionar problemas de coordinación que dificulten el logro de acuerdos que permitan la correcta asignación de los activos hacia las oportunidades de inversión más rentables (Indro et al., 1999) ${ }^{(21)}$. También otras variables de control resultan significativas en las estimaciones realizadas, como el tipo de sistema financiero (SF) o las dummy temporales (YEAR), estas últimas analizadas de manera global a través del test de Wald de significación conjunta. Por lo que se refiere al resto de las variables, destaca la ausencia de significación del nivel de endeudamiento (DT/PT) y de la probabilidad de quiebra (Z). Estos resultados pueden venir explicados por la propia composición de la muestra, empresas insolventes, en las que el rendimiento del mercado no parece verse afectado por su situación relativa de solvencia. Al tratarse de empresas con dificultades financieras las variables relacionadas con sus posibilidades de continuidad ejercen una influencia significativa, mientras que el mercado no parece reaccionar ante su situación financiera anterior.

Por lo que se refiere a la interpretación de los coeficientes estandarizados, de todas las variables incluidas, los índices de eficiencia de la legislación concursal se muestran como las variables con mayor influencia. Esto pone de manifiesto la relevancia que la normati-

(21) La relación negativa entre el tamaño y la rentabilidad de las acciones ha sido encontrada incluso fuera del contexto de las empresas con problemas de insolvencia (Fama y French, 1992). 
va de insolvencias tiene sobre el comportamiento del valor de mercado de las empresas en dificultades financieras, con una importancia superior a las propias características de las empresas.

\section{CONCLUSIONES}

El propósito de este trabajo ha sido aportar la perspectiva de la valoración del mercado al debate acerca del desarrollo de un procedimiento concursal óptimo, considerando como factor fundamental el grado de eficiencia que esta elección supone. En este sentido, además de considerar la eficiencia en términos ex ante y ex post, se ha centrado la atención en la importancia que pueden tener las medidas que afectan a la eficiencia intermedia.

Por lo tanto, se ha tratado de evaluar no sólo la orientación de la legislación sino también el objetivo perseguido por la legislación con las medidas concretas establecidas en el procedimiento, y su repercusión sobre el valor de mercado de la empresa. En este sentido se analizan las características de las legislaciones concursales que tratan de mejorar la eficiencia ex ante y ex post de todo el procedimiento, a través del estudio del efecto que los mecanismos establecidos en la legislación ejercen sobre ambos tipos de eficiencia, de la valoración que el mercado realiza de esas medidas, y del grado de eficiencia logrado con ellas, centrando la atención, exclusivamente, en las implicaciones que tienen para las empresas que se enfrentan a problemas de insolvencia. Para ello, se han desarrollado dos índices que permiten cuantificar el grado de eficiencia de las regulaciones, lo que resulta imprescindible a la hora de abordar un estudio de estas características.

Los resultados muestran que las legislaciones que establecen fuertes incentivos para un comportamiento eficiente ex ante provocan una mayor pérdida de valor en las empresas que se encuentran en dificultades financieras, al producirse mayores costes que empeoran la eficiencia intermedia. Esto origina que las medidas orientadas a mejorar la eficiencia ex ante tengan una repercusión negativa si consideramos su influencia únicamente sobre las empresas que atraviesan problemas de insolvencia, desde el momento en que se empiezan a manifestar sus dificultades hasta que se realiza la declaración legal de insolvencia.

En el caso de la eficiencia ex post, los resultados muestran una valoración positiva de este tipo de mecanismos incluidos en el procedimiento para las empresas con mayores probabilidades de éxito, y una influencia negativa en el caso de empresa con peores perspectivas de futuro. De esta forma, la valoración positiva no se produce en el caso de legislaciones que favorecen la continuidad de todas las empresas, sino que aparece para aquellos mecanismos que persiguen lograr la eficiencia ex post del proceso permitiendo el mantenimiento de las empresas cuyo valor percibido por el mercado es superior a su valor de liquidación.

Por lo tanto, las medidas establecidas para mejorar la eficiencia ex ante pueden tener un efecto negativo sobre las empresas que tienen problemas de insolvencia antes de la entrada en el procedimiento concursal, hecho que debería ser considerado al fijar los 
objetivos que se pretenden lograr con el establecimiento de determinadas medidas concretas, evitando así la aparición de efectos negativos indirectos sobre las empresas con dificultades financieras. Por otro lado, también se pone de manifiesto la importancia de establecer mecanismos que permitan la continuidad de las empresas económicamente viables, mediante la existencia de procedimientos que faciliten la negociación de los distintos agentes implicados, que eviten liquidaciones que resulten ineficientes desde un punto de vista económico.

Estos resultados aportan un elemento más a la discusión sobre cuál es el sistema concursal más adecuado, que puede resultar de interés a la hora de fijar los objetivos que se pretenden lograr, y de disponer de los mecanismos adecuados para su consecución, estableciendo, de manera inequívoca, sus efectos sobre todas las empresas, y evitando el establecimiento de medidas concretas de carácter opuesto, que dificulten alcanzar una salida eficiente del procedimiento concursal. Una visión parcial, que sólo se centre en el tratamiento de las empresas una vez que han entrado en el procedimiento concursal, podría tener los efectos contrarios a los deseados, por sus implicaciones para las empresas antes de la entrada en el procedimiento.

Las conclusiones que se derivan de este trabajo son especialmente relevantes en el momento actual, con las reformas que se han producido en la ley de quiebras en distintos países. Tanto la reforma de Alemania (1999), España (2004) y especialmente la del Reino Unido (2003) han supuesto una reducción de la protección de los acreedores y un mayor sesgo hacia la continuidad de la empresa. En este sentido, parece que el objetivo de maximizar la eficiencia ex ante se deja en un segundo lugar frente al tratamiento de las empresas que de hecho atraviesan por problemas de insolvencia. No obstante, los efectos que estas reformas recientes pueden producir no se recogen en este trabajo, puesto que su inclusión en el mismo podrían introducir distorsiones en los resultados obtenidos, tal y como se detalla en la descripción de la muestra. No obstante sería interesante, una vez que se disponga de un periodo muestral suficiente para evitar sesgos, realizar una comparativa para analizar el efecto real que esas modificaciones introducen en las conclusiones alcanzadas, lo que sin duda aportará una evidencia más robusta acerca del problema analizado.

\section{BIBLIOGRAFÍA}

Agarwal, V., y Taffler, R. 2008. Comparing the performance of market-based and accountingbased bankruptcy prediction models. Journal of Banking \& Finance 32: 1.541-1.551.

Aghion, P.; Hart, O., y Moore, J. 1992. The economics of bankruptcy reform. Journal of Law, Economics and Organization 8 (3): 523-546.

Aharony, J.; Jones, C., y Swary, I. 1980. An analysis of risk and return characteristics of corporate bankruptcy using capital market data. Journal of Finance 35 (4): 1.001-1.016.

Alderson, M., y BETKER, B. 1999. Assessing post-bankruptcy performance: an analysis of reorganized firms cash flows. Financial Management 28 (2): 68-82.

Altman, E., 1968. Financial ratios, discriminant analysis and the prediction of corporate bankruptcy. Journal of Finance 23 (4): 589-609. 
Asquith, P.; Gertner, R., y Scharfstein, D. 1994. Anatomy of financial distress: an examination of junk-bond issuers. The Quarterly Journal of Economics 109 (3): 625-658.

BAE, K., y GoYAL, V. 2004. Property rights protection and bank loan pricing. Working paper: Queen's University.

BAIRD, D. 1991. The initiation problem in bankruptcy. International Review of Law and Economics 11: 223-232.

BARBER, B., y Lyon, J. 1997. Detecting long run abnormal performance stock returns: the empirical power and specification of test statistics. Journal of Financial Economics 43: 341-372.

BEAVER, W. 1968. Market prices, financial ratios and the prediction of failure. Journal of Accounting Research 6 (2): 179-192.

Berkovitch, E., e Israel, R. 1999. Optimal Bankruptcy laws across different Economic systems. The Review of Financial Studies 12 (2): 347-377.

BETKER, B. 1995. Management incentives, equity's bargaining power, and deviations from absolute priority in Chapter 11 bankruptcies. Journal of Business 68 (2): 161-183.

Blazy R.; Weill, L., y Petey, J. 2009. Can Bankruptcy Codes Create Value? Evidence from Creditors' Recoveries in France, Germany, and the UK. European Financial Management Association 2010 Annual Meetings.

Brav, A.; GeCZy, C., y Gompers, P. 2000. Is the abnormal return following equity issuances anomalous?. Journal of Financial Economics 56: 209-249.

BRIs, A.; Welch, I., y Zhu, N. 2006. The cost of Bankruptcy: Chapter 7 liquidation versus Chapter 11 reorganization. Journal of Finance 61 (3): 1.253-1.303.

Brown, D. 1996. Corporate rescue: Insolvency law in practice. John Wiley \& Sons.

CAMPBelL, D. 1992. International corporate insolvency law. Butterworths.

Chatterjee, S.; Dhillon, U., y Ramírez, G. 1996. Resolution of financial distress: debt restructurings via Chapter 11, pre-packaged bankruptcies and workouts. Financial Management 25 (1): 5-18.

- 2004. Debtor in possession financing. Journal of Banking and Finance 28 (12): 3.097-3.111.

Chopard, B.; Guigou, J.; Fimayer, A., y Blazy, R. 2007. Financial Versus Social Efficiency of Corporate Bankruptcy Law: The French Dilemma?. 3rd Annual Conference on Empirical Legal Studies Papers.

Clark, T., y Weinstein, M. 1983. The behaviour of the common stock of bankrupt firms. Journal of Finance 38 (2): 489-504.

Cornelui, F., y Feldi, L. 1997. Ex-ante efficiency of bankruptcy procedures. European Economic Review 41: 475-485.

Couwenkerg, O. 2001. Survival rates in bankruptcy systems: overlooking the evidence. European Journal of Law and Economics 12: 253-273.

Cowan, A., y Sergeant, A. 2001. Interacting biases, non-normal return distributions and the performance of tests for long-horizon event studies. Journal of Banking and Finance 25: 741-765.

Dahiya , S.; John , K.; Puri, M., y Ramírez, G., 2003. Debtor-in-Possession financing and bankruptcy resolution: empirical evidence. Journal of Financial Economics 69 (1): 259-280.

Davydenko, S., y Franks, J. 2008. Do Bankruptcy Codes Matter? A Study of Defaults in France, Germany and the UK. Journal of Finance 63 (2): 565-608.

Dawkins, M., y Rose-Green, E. 1998. Prior Wall Street Journal announcements of possible bankruptcy filings and price reactions to subsequent bankruptcy filings. Journal of Business, Finance and Accounting 25 (7\&8): 813-827. 
Delaney, K. 1992. Strategic bankruptcy. How corporations and creditors use Chapter 11 to their advantage. Los Angeles: University of California Press.

Djankov, S.; McLiesh, C., y Shleifer, A. 2007. Private credit in 129 countries. Journal of Financial Economics 84: 299-329.

Eberhart, A.; Moore, W., y Roenfeldt, L. 1990. Security pricing and deviations from the absolute priority rule in bankruptcy proceedings. Journal of Finance 45 (5): 1.457-1.469.

Elayan, F., y Meyer, T. 2001. The impact of receiving Debtor-in-possession financing on the probability of successful emergence and time spent under Chapter 11 bankruptcy. Journal of Business, Finance and Accounting 28 (7\&8): 905-942.

EspinA, A. 1999. La reforma del derecho concursal y la eficiencia económica. Madrid: Consejo General de Colegios de Economistas de España.

Fama, E., y French, K. 1992. The cross section of expected stock returns. Journal of Finance 47: 427-465.

- 1998. Market efficiency, long-term returns, and the behavioural finance. Journal of Financial Economics 49: 283-306.

Fama, E. F., y French, K. R. 1992. The Cross-Section of Expected Stock Returns. Journal of Finance 47 (2): 427-465.

Ferris, S.; Jayaraman, N., y MakhiJa, A. 1996. The Impact of Chapter 11 Filings on the Risk and Return of Security Holders, 1979-1989. Advances in Financial Economics 2: 93-118.

Franks, J., y Torous, W. 1994. A comparison of financial recontracting in distressed exchanges and Chapter 11 reorganizations. Journal of Financial Economics 35: 349-370.

Franks, J.; Nyborg, K., y Torous, W. 1996. A comparison of US, UK and German insolvency codes. Financial Management 25(3): 86-101.

Garcia Villaverde, R. 1993. Textos de derecho concursal europeo, Madrid: Editorial Dykinson.

Gertner, R., y Scharfstein, D. 1991. A theory of workouts and the effects of reorganization law. Journal of Finance 46(4): 1.189-1.222.

Gilson, S.; John, K., y Lang, H. P. 1990. Troubled debt restructurings: An empirical study of private reorganization of firms in default. Journal of Financial Economics 27: 315-353.

González Méndez, V. M., y GonzÁlez Rodríguez, F. 2000. Procedimientos de resolución de insolvencia financiera en España: costes de insolvencia y transferencias de riqueza. Investigaciones Económicas 24(2): 357-384.

HaRT, O. 2000. Different approaches to bankruptcy. Harvard Institute of Economic Research Discussion Paper 1903.

Holmstrom, B., y Myerson, R. 1983. Efficient and durable decision rules with incomplete information. Econometrica 51(6): 1.799-1.819.

Indro, D.; Leach, R., y LeE, W. 1999. Sources of gains to shareholders from bankruptcy resolution. Journal of Banking and Finance 23: 21-47.

Jakobsen, J., y Voetmann, T. 2005. A New Approach for Interpreting Long-Run Returns, Applied to IPO and SEO Stocks. Annals of Economics and Finance 6: 337-363.

Jayaraman, N.; Sabherwal, S., y Shrikhande, M. 2001. Do country specific bankruptcy codes determine long-term financial performance? The case of Metallgesellschaft AG and Columbia Gas System. Journal of International Financial Management and Accounting 12(2): 188-224.

Jensen, M., y Meckling, W., 1976. Theory of the firm: Managerial behavior, agency cost, and ownership structure. Journal of Financial Economics 3: 305-360. 
Jiménez Cardoso, S. 1996. Una evaluación crítica de la investigación empírica desarrollada en torno a la solvencia empresarial. Revista Española de Financiación y Contabilidad 26(87): 459-479.

KAISER, K. 1996. European bankruptcy laws: implications for corporations facing financial distress. Financial Management 25(3): 67-85.

Kothari, S., y Warner, J. 1997. Measuring long-horizon security price performance. Journal of Financial Economics 43: 301-339.

La Porta, R., y Lopez de Silanes, F. 2001. Creditor protection and bankruptcy law reform. En Claessens, S.; Duankov, S., y Mody, A. Eds. Resolution of financial distress. An international perspective on the design of bankruptcy laws, Washington: World Bank Institute Development Studies: 65-90.

La Porta, R.; Lopez de Silanes, F.; Shleifer, A., y Vishny, R. 1998. Law and Finance. Journal of Political Economy 106: 1.113-1.156.

Lasfer, M. A.; Sudarsaman, P., y Taffler, R. 1996. Financial Distress, Asset Sales, and Lender Monitoring. Financial Management 25(3): 57-66.

Levine, R. 2002. Bank-based or Market-based Financial Systems: Which is Better? Journal of Financial Intermediation 11: 398-428.

López GutiérRez, C.; Torre Olmo, B., y Díaz Díaz, B. 2005. Características determinantes y contenido informativo de los mecanismos de reestructuración financiera. Revista Europea de Dirección y Economía de la Empresa 14(2): 111-124.

López Gutiérrez, C.; García Olalla, M., y Torre Olmo, B. 2009. The influence of bankruptcy law on equity value of financially distressed firms: A European comparative analysis. International Review of Law and Economics 29: 229-243.

Loughran, T., y Ritter, J. 1995. The New Issues Puzzle. Journal of Finance 50(1): 23-51.

Lutтiкнus, K. 2008. The effectiveness and efficiency of corporate insolvency law. Part 1. International Insolvency Review 17(3): 189-209.

Lyon, J.; BARBER, B., y TsaI, C. 1999. Improved methods for tests of long-run abnormal stock returns. Journal of Finance 54(1): 165-201.

MокAL, R. J. 2004. Administrative Receivership and Administration: an analysis. Current Legal Problems 57: 355-392.

Mora Enguídanos, A. 1995. Utilidad de los modelos de predicción de la crisis empresarial. Revista Española de Financiación y Contabilidad 24(83): 281-300.

Morgado, A., y Pindado, J., 2003. The underinvestment and overinvestment hypotheses: an analysis using panel data. European Financial Management 9(2): 163-177.

Pindado, J.; Rodrigues, L., y De la Torre, C. 2008. How do insolvency codes affect a firm's investment? International Review of Law and Economics 28: 227-238.

Pistor, K.; Raiser, M., y Gelfer, S. 2000. Law and Finance in Transition Economies. Economics of Transition 8: 325-368.

Povel, P., 1999. Optimal «Soft» or «Tough» bankruptcy procedures. Journal of Law, Economics, and Organization 15(3): 659-684.

Qian, J., y Strahan, P. 2007. How law and institutions shape financial contracts: The case of bank loans. Journal of Finance 62(6): 2.803-2.834.

RAJAK, H.; HoRrocks, P., y Bannister, J. A. 1995. European corporate insolvency: a practical guide. John Wiley \& Sons. 
Rimbey, J.; Anderson, S., y Born, J. 1995. Shareholder wealth responses to bankruptcy filing announcements under the Chandler and Reform Acts. The Financial Review 30(1): 1-22.

RitTer, J. 1991. The Long-Run Performance of Initial Public Offerings. Journal of Finance 46(1): 3-27.

Rose-Green, E., y Dawkins, M. 2002. Strategic bankruptcies and price reactions to bankruptcy filings. Journal of Business, Finance and Accounting 29(9\&10): 1.319-1.335.

Sмгтн, D. C., у Sтrömвerg, P. 2005. Maximizing the value of distressed assets: Bankruptcy law and the efficient reorganization of firms. En Honohan, P., y Laeven, L. Eds. Systemic financial crisis. Containment and resolution, Cambridge: Cambridge University Press: 232-275.

STILPON, N. 1994. Corporate bankruptcy and reorganization procedures in OECD and central and eastern European Countries. Centre for co-operation with the EC.

Uhrig-Homburg, M. 2005. Cash-flow shortage as an endogenous bankruptcy reason. Journal of Banking \& Finance 29: 1.509-1.534.

WЕвв, D. 1991. An economic evaluation of insolvency procedures in the United Kingdom: Does the 1986 insolvency act satisfy the creditors' bargain? Oxford Economics Papers 43: 139-157.

Wніте, H. 1980. A heteroscedasticity-consistent covariance matrix estimator and a direct test for heterocedasticity. Econometrica 48: 817-838.

Wнгте, M. J. 1989. The corporate bankruptcy decision. Journal of Economics Perspectives 3: 129-151.

- 1996. The cost of corporate bankruptcy: an US-European comparison. En Bhandari, J., y WEISS, L. Eds. Corporate bankruptcy. Economic and legal perspectives, Cambridge: Cambridge University press: 467-500.

Wruck, K. 1990. Financial distress, reorganization, and organizational efficiency. Journal of Financial Economics 27: 419-444. 


\begin{tabular}{|c|c|c|c|c|c|c|c|c|c|c|}
\hline \multirow{7}{*}{ 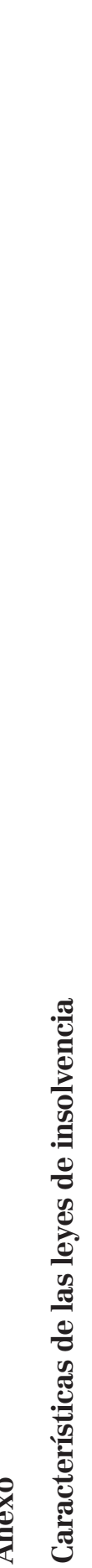 } & 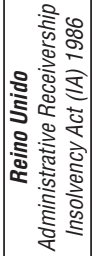 & 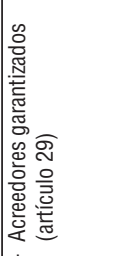 & 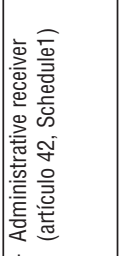 & 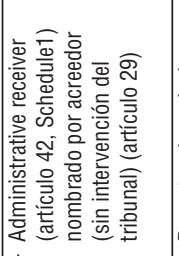 & 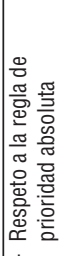 & 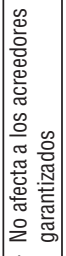 & & 은 & 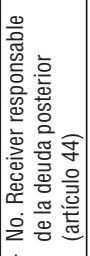 & \multirow{7}{*}{ 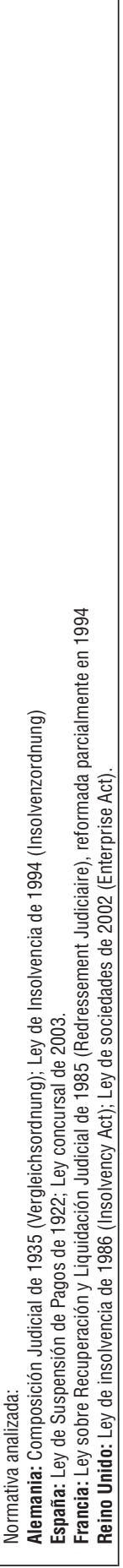 } \\
\hline & 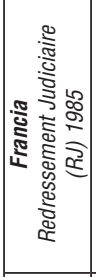 & 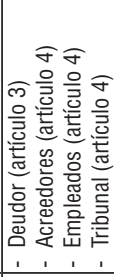 & 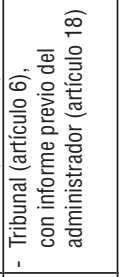 & 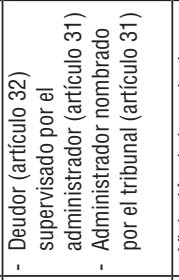 & 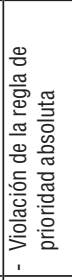 & & 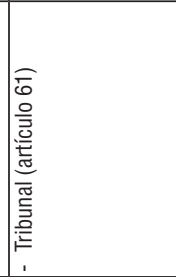 & 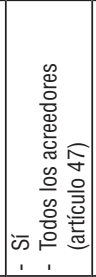 & 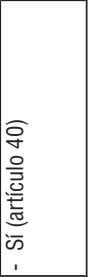 & \\
\hline & 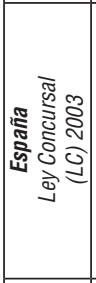 & 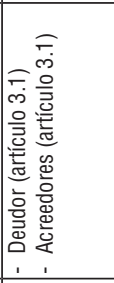 & 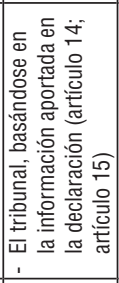 & 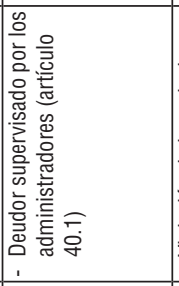 & 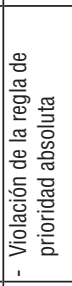 & & 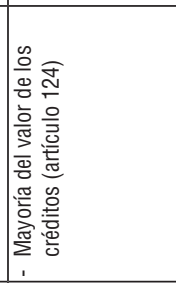 & 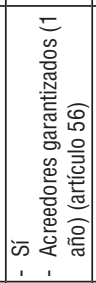 & 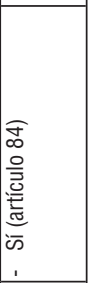 & \\
\hline & 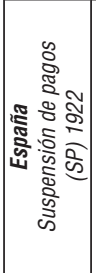 & 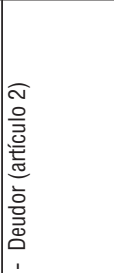 & 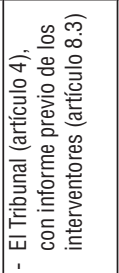 & 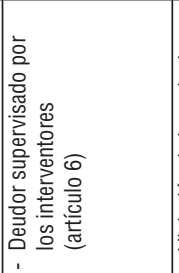 & 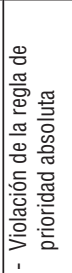 & 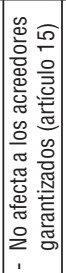 & 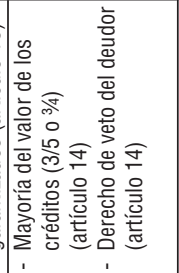 & 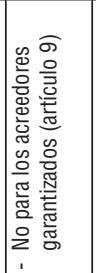 & 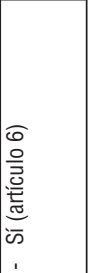 & \\
\hline & 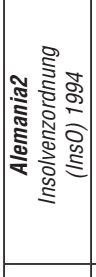 & 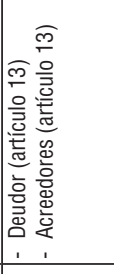 & 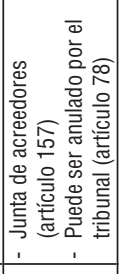 & 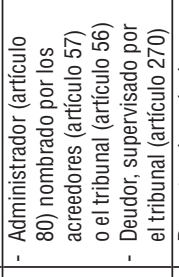 & 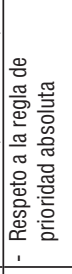 & & 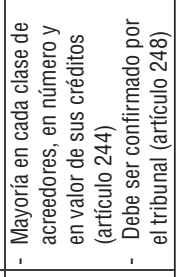 & 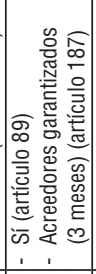 & 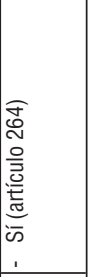 & \\
\hline & 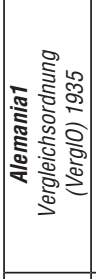 & 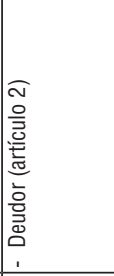 & 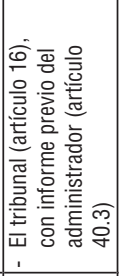 & 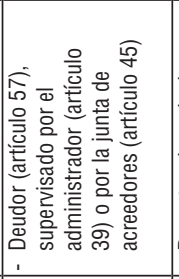 & 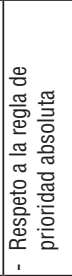 & 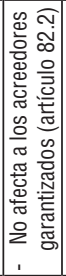 & 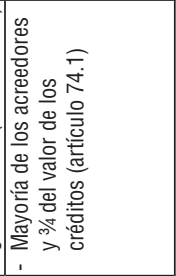 & 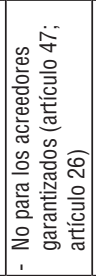 & 2 & \\
\hline & & 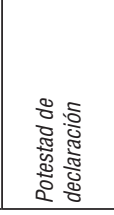 & 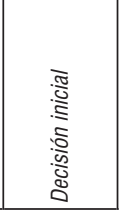 & 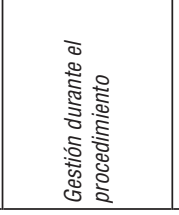 & 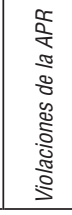 & 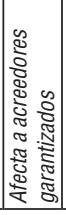 & $\begin{array}{l}\frac{\pi}{2} \\
\frac{0}{2} \\
\frac{2}{0} \\
\frac{0}{2} \\
\frac{\pi}{0} \\
\frac{0}{2} \\
\end{array}$ & 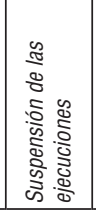 & 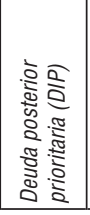 & \\
\hline
\end{tabular}

